\title{
Interactive comment on "How vadose zone mass and energy transfer physics affects the ecohydrological dynamics of a Tibetan meadow?" by Lianyu Yu et al.
}

\section{Lianyu Yu et al.}

yulianyu257@gmail.com

Received and published: 31 July 2020

We thank the reviewer very much for the constructive comments. We made the point by point response to the comments. The reviewers' comments are in black fonts indexed by numbers. Our response is in blue fonts and red fonts are the updates in the manuscript.

General Comment: "How vadose zone mass and energy transfer physics affects the ecohydrological dynamics of a Tibetan meadow?" by Yu et al. addresses the importance of including freezing and thawing processes and coupling water and energy 
balance equations of vadose zone models to simulate ecohydrological dynamics. The authors use data from a high-elevation meadow of the Tibetan plateau to evaluate the outcomes of their different simulations. From their results, the authors claim that adding model complexity improves the model estimates of soil temperature and moisture but may not be necessary to model vegetation dynamics in these regions.

The manuscript is generally well written and understandable. While the presentation and interpretation of the results makes sense, it sometimes lacks clarifications and could benefit from a deeper analysis. I think the model, even fully coupled, does a poor job at reproducing ice content and thermal conditions of deeper layers. As pointed by the authors, some data collected to evaluate the different simulations seem wrong, while others are simply missing, which means it can be barely used to validate any of the simulations. However, the conclusions of this manuscript remain valid and of considerable interest and is valuable to anyone wondering about the importance of including dynamic freezing and thawing processes in their models. Overall, this manuscript has the potential to be an interesting and useful contribution to The Cryosphere, but some critical issues, mostly in the methodology section, need to be addressed before considering publication.

I also think this manuscript does not reach its full potential. It could use various level of complexity in their approach to include freezing and thawing processes. For example, the CPLD simulation could be divided into different simulations: one that only considers latent heat, one that only considers the effect of ice content on the hydraulic conductivity, etc. This would show modelers which component of the freezing/thawing processes is important to consider, and which one is potentially not. However, I acknowledge that this suggestion would involve substantial additional work, so I understand if the authors would rather not make this change.

Response: Many thanks for your constructive comments. We made modifications of the methodology section, in which the clear writing flow was followed. The governing equations and main constitutive equations for different models were clearly presented. The differences among three models regarding soil physical processes were illustrated

Printer-friendly version

Discussion paper 
in Table 1 and Sect. 3.5. The constitutive equations of unfrozen water content, temperature dependence of liquid flow, the ice effect on hydraulic conductivity, and water vapor density was presented in supplement material (Section S1). We added the geographical location of the Maqu soil moisture and temperature monitoring network and the central experimental site (Figure 1, thus the original figure number was changed). In addition, the description of data gaps was briefly presented. We appreciate your suggested simulations on investigating which component of the freezing/thawing processes is important. This question indeed can be investigated in our future work. We would rather not making this change right now though, and want to present the current results step by step to avoid overwhelming information in one manuscript.

1. Introduction: Well written and introduce well the topic and the problematic. However, I think the authors could add a few lines about the efforts done so far to model freezing and thawing processes in coupled water \& energy models for cold regions. There are numerous subsurface and surface models already doing that. They do not all necessarily simulate ecological dynamics, but some studies have provided useful information about the impact of neglecting freezing dynamics.

Response: Thanks a lot for your suggestions. We added in the first part of Introduction some studies that evaluate the modeling of freezing and thawing processes coupled water and energy and pointed out that the novelty here is that also ecohydrological dynamics are jointly evaluated.

Changes in the manuscript: "Concurrently, researchers developed dedicated models, e.g, SHAW (Flerchinger and Saxton, 1989), HYDRUS (Hansson et al., 2004), MarsFlo (Painter, 2011), and STEMMUS-FT (Yu et al., 2018), considering the soil water and heat coupling physics for frozen soils. Promising simulation results have been reported for the soil hydrothermal regimes. While these efforts mainly focus on understanding the surface and subsurface soil water and heat transfer process (Yu et al., 2018) and stress the role of physical representation of freezing/thawing process (Boone et al., 2000;Wang et al., 2017b;Zheng et al., 2017), they seldomly take into account the

Printer-friendly version

Discussion paper

\section{3}


2. L56: Unclear what is meant by "changes of frozen ground". Maybe the authors meant something like "variations in seasonally frozen ground thickness"?

Response: Yes, here it is meant to be "variations in seasonally frozen ground thickness". Corrected in the manuscript.

\section{L58: What are those divergences? Please provide a few examples.}

Response: We added some examples here.

Changes in the manuscript: "However, there are divergences with regard to the expected ecosystem changes across the Tibetan Plateau (Cheng and Wu, 2007; Qin et al., 2016;Wang et al., 2018;Zhao et al., 2010). In response to climate warming, the degradation of frozen ground can positively affect the vegetation growth in Tibetan Plateau mountainous region (Qin et al., 2016), but it can also lead to degradation of grasslands (Cheng and $\mathrm{Wu}, 2007)$, depending on soil hydrothermal regimes and climate conditions (Qin et al., 2016;Wang et al., 2016)."

4. L70: I am not sure "complexity" is the best word here. According to the authors, they are testing their models with or without freezing dynamics, and with or without water and energy coupling. As I understand it, there are no different "complexities" in the way frozen soil is represented or in the way the coupling is achieved.

Response: Here we intended to say that the vadose zone water and heat transfer physics is represented in an increasingly complex way (from the T\&C without the freezing dynamics, with freezing dynamics but uncoupled water and heat transfer, to the coupling of T\&C with STEMMUS to account for fully coupled processes). For unCPLD model (T\&C), soil water and heat are independently simulated, ice effect is not considered. For the unCPLD-FT model (T\&C-FT), the ice effect on hydraulic conductivity is considered via an impedance factor; thermal conductivity and capacity are affected by the ice content; latent heat change during the freezing/thawing period is 
taken into account but heat does not exchange with liquid water. During the non-frozen period, soil water and heat are still independently transferred. For the CPLD model (T\&C-STEMMUS), the ice effect on the hydrothermal properties is considered; latent heat change from the freezing/thawing process is also taken into account. Vapor flow, which links the soil water and heat flow, is simulated. The thermal effect on water flow is explicitly considered. Thus, soil water and heat transfer processes are tightly coupled not only during the frozen period but also during the non-frozen period for the CPLD model. We better explained these aspects in Sect. 3.5.

Changes in the manuscript: "To investigate the role of increasing complexity of vadose zone physics in ecosystem functioning, three numerical experiments were designed on the basis of the aforementioned modeling framework (Table 1). First experiment, the T\&C original model was run as stand-alone, termed as unCPLD simulation. For the unCPLD model, soil water and heat transfer is independent with no explicit consideration of soil ice effect. The second experiment, the updated T\&C model with explicit consideration of freezing/thawing process was run as it can estimate the dynamics of soil ice content and the related effect on water and heat transfer (e.g., blocking effect on water flow, heat release/gain due to phase change) but otherwise being exactly equal to T\&C original model. This second simulation is named the unCPLD-FT simulation, where the term unCPLD generally refers to the fact that T\&C model and STEMMUS model are not yet coupled. The third experiment, STEMMUS model was coupled with T\&C model to enable not only frozen soil physics but also additional processes and most importantly the tight coupling of water and heat effects. This simulation is named CPLD simulation. In this third scenario, vapor flow, which links the soil water and heat flow, is explicitly considered. In addition to the ice blocking effect as presented in unCPLD-FT, the thermal effect on water flow is also expressed with the temperature dependence of hydraulic conductivity and matric potential. Furthermore, not only the latent heat due to phase change, but also the convective heat due to liquid/vapor flow is simulated." 
5. Section 2.1: It is unclear until the reader reaches the results section if the experimental site is underlain by permafrost or not. Please add somewhere in this section that the site only has seasonally frozen ground.

Response: We added this information in the text in Sect. 2.1 as "Seasonally frozen ground is characteristic of this site, with the maximum freezing depth approaching around $0.8 \mathrm{~m}$ under current climate conditions."

6. L 93: It is written here that the SMST profiles are measuring temperature and soil moisture at a depth of $80 \mathrm{~cm}$. However, figures from the results section are never showing data at $80 \mathrm{~cm}$, only at $60 \mathrm{~cm}$ (which is not listed in line 93). Please fix or clarify.

Response: For Maqu soil moisture and soil temperature (SMST) monitoring network, the SMST profiles are generally measured at depths of $5 \mathrm{~cm}, 10 \mathrm{~cm}, 20 \mathrm{~cm}, 40 \mathrm{~cm}$, and $80 \mathrm{~cm}$. In addition, we have installed some additional $5 \mathrm{TM} \mathrm{ECH2O} \mathrm{probes} \mathrm{to} \mathrm{enrich}$ the SMST profile information at specific points. Here, the additional SMST profiles, installed at $2.5 \mathrm{~cm}, 5 \mathrm{~cm}, 10 \mathrm{~cm}, 20 \mathrm{~cm}, 40 \mathrm{~cm}, 60 \mathrm{~cm}$, and $100 \mathrm{~cm}$, were employed for validating the model simulations. We clarified this point in Sect. 2.1.

Changes in the manuscript: "A few dedicated SMST profiles, with sensors installed at depths of $2.5 \mathrm{~cm}, 5 \mathrm{~cm}, 10 \mathrm{~cm}, 20 \mathrm{~cm}, 40 \mathrm{~cm}, 60 \mathrm{~cm}$, and $100 \mathrm{~cm}$, were used for validating the model simulations."

7. Figure 1b: If the figure is indeed showing both freezing and thawing fronts, I would recommend using different colors. It is unclear which zone is thawed and which one is frozen (mostly in 2017-2018). I also think the y-axis should not reach $100 \mathrm{~cm}$. Based on the text, there is no sensor deeper than $80 \mathrm{~cm}$. If I understood correctly, the graph is plotting information the authors do not have. Please fix or clarify in the text. Furthermore, is the data here interpolated from the 5 sensors described in line 93 ?

Response: Different colors are now presented in Figure $2 b$, with the freezing fronts

Printer-friendly version

Discussion paper
Interactive

comment 
color blue and thawing fronts color red. In the updated Figure $2 \mathrm{~b}$, soil temperature measurements at depths of $2.5 \mathrm{~cm}, 5 \mathrm{~cm}, 10 \mathrm{~cm}, 20 \mathrm{~cm}, 60 \mathrm{~cm}$, and $100 \mathrm{~cm}$ were used to generate the freezing/thawing front propagation dynamics. Soil temperature measurements at $40 \mathrm{~cm}$ are not used as its large data gaps during 2017-2018 wintertime (see Table R.1).

Table R.1. The main data gaps for soil moisture/soil temperature (SMST) measurements

\begin{tabular}{ll}
\hline SMST & Data gaps \\
\hline $2.5 \mathrm{~cm}$ & $3 / 25 / 2016-06 / 08 / 2016,3 / 29 / 2017-27 / 07 / 2017$ \\
$5 \mathrm{~cm}$ & $3 / 25 / 2016-06 / 08 / 2016,3 / 29 / 2017-27 / 07 / 2017$ \\
$10 \mathrm{~cm}$ & $3 / 25 / 2016-06 / 08 / 2016,3 / 29 / 2017-27 / 07 / 2017$ \\
$20 \mathrm{~cm}$ & $3 / 25 / 2016-06 / 08 / 2016,3 / 29 / 2017-27 / 07 / 2017$ \\
$40 \mathrm{~cm}$ & $3 / 25 / 2016-06 / 08 / 2016,3 / 29 / 2017-12 / 08 / 2018$ \\
$60 \mathrm{~cm}$ & $3 / 25 / 2016-06 / 08 / 2016,3 / 29 / 2017-27 / 07 / 2017$ \\
$100 \mathrm{~cm}$ & $3 / 25 / 2016-06 / 08 / 2016,3 / 29 / 2017-27 / 07 / 2017$ \\
\hline
\end{tabular}

Changes in the manuscript: "A few dedicated SMST profiles, with sensors installed at depths of $2.5 \mathrm{~cm}, 5 \mathrm{~cm}, 10 \mathrm{~cm}, 20 \mathrm{~cm}, 40 \mathrm{~cm}, 60 \mathrm{~cm}$, and $100 \mathrm{~cm}$, were used for validating the model simulations. Note that there are data gaps (25th Mar -8 th June, 2016; 29th Mar - 27th July, 2017, extended to 12th Aug, 2018 for $40 \mathrm{~cm}$ ) due to the malfunction of instruments and the difficulty to maintain the network under harsh environment."

8. L 124: Please provide more information about the missing data. When and where? This should also be shown in Figure 1b. For example, the authors can use a greyed area to show where data are missing. This is crucial considering the authors are using this data or comparison purposes in Figure 6.

Response: The main data gaps for SMST measurements are summarized as Table 
R.1. For Figure 1b, the propagation of freezing/thawing front was obtained by interpolated the SMST profile measurements $(2.5 \mathrm{~cm}, 5 \mathrm{~cm}, 10 \mathrm{~cm}, 20 \mathrm{~cm}, 60 \mathrm{~cm}$, and 100 $\mathrm{cm})$. Here, soil temperature measurements at a depth of $40 \mathrm{~cm}$ were omitted as its long data gaps during the second winter period (2017-2018).

Changes in the manuscript: "Note that there are data gaps (25th Mar - 8th June, 2016; 29th Mar - 27th July, 2017, extended to 12th Aug, 2018 for $40 \mathrm{~cm}$ ) due to the malfunction of instruments and the difficulty to maintain the network under harsh environment."

9. Equation 1: Check units. Are the units of $S$ really s-1 or rather $\mathrm{kg} \mathrm{m}-3 \mathrm{~s}-1$ ?

Response: The unit of $\mathrm{S}$, the sink term, is $\mathrm{kg} \mathrm{m}-3 \mathrm{~s}-1$ with the consideration of water density. We corrected the unit of $S$ as ' $k g$ m-3 s-1'.

10. Equation 2: Undefined variables: $\mathrm{dz}, \mathrm{rH}, \mathrm{rL}, \mathrm{nc}$

Response: Equation 2: Note that to avoid confusion, the terms not used in this simulation case was deleted from Eq. 2. We added the relevant description.

Changes in the manuscript: $d_{z, i} \frac{d \theta_{i}}{d t}=q_{i-1}-q_{i}-T_{v} r_{v, i}-E_{s}-E_{b a r e}$ (2)

where $d_{z, i}(\mathrm{~m})$ is the thickness of layer $\mathrm{i} ; q_{i}\left(\mathrm{~ms}^{-1}\right)$ is the vertical outflow from a layer $\mathrm{i} ; T_{v}\left(\mathrm{~ms}^{-1}\right)$ is the transpiration fluxes from the vegetation; $r_{v, i}$ is the fraction of root biomass contained in soil layer $\mathrm{i}$; $E_{\text {bare }}\left(\mathrm{ms}^{-1}\right)$, evaporation from the bare soil; $E_{s}$ $\left(m s^{-1}\right)$, evaporation from soil under the canopy.

11. Equation 4: Csoil is presented as the specific heat capacity of the bulk soil, but I think it is the volumetric heat capacity. The units do not match otherwise. The same issue arises in equations 6, 7 and 8. Equation 8 is from Hansson et al. (2004), where Csoil is defined as the volumetric heat capacity.

Response: Many thanks for pointing out this. Equation 4: There was an inconsistency between the left and right side of Equations 4, 6, 7, and 8 (now Eqs. 3, 4, 5, 6). We multiplied Csoil with the soil density $\rho_{\text {soil }}$ to make it consistent. Capp was clarified as 
the apparent volumetric heat capacity here as Equation 8 (now Eq. 5).

12. Equation 8: Please define which phase is represented by $d \theta$. It should be liquid water.

Response: Thanks a lot for your comment. We added some text as "differential (specific) water capacity $d \theta / d \varphi$ at a given liquid water content $\theta$ ".

Interactive

comment

13. Section 2.4.4: The authors describe in detail the equations of the two models but do not explain the critical components of the added freezing/thawing processes, except for latent heat. They do provide references but, considering that this is supposedly an important aspect of this manuscript, they should at least describe in more details the different equations used. For example, the calculation of the hydraulic conductivity for a frozen medium is quite important. The authors refer here to Hansson et al. (2004), which uses an impedance factor. This method is widely used, but the impedance factor is an arbitrary number that is likely to change based on the type of soil. The authors never write which impedance factor is used. I am also concerned that the authors are using the method from Dall'Amico et al. (2011) for the soil freezing characteristic curve and the method from Hansson et al. (2004) for the apparent heat capacity. These two papers both use a form of the Clausius-Clapeyron equation with the van Genuchten model, but with different approaches. I could be wrong but, depending on how they have been used, these two methods may not be compatible with each other. More information is required here to make sure the freezing model used by the authors is valid.

Response: We added the description of the freezing/thawing process, i.e., how unfrozen water content, hydraulic conductivity is calculated, the assignment of impedance factor, temperature dependency of hydraulic conductivity/matric potential, vapor density parameterization. As this information does not represent the main physical equation but rather parameterizations, we did not list them in the main text but in the supplemental materials (Supplement S1).

Printer-friendly version

Discussion paper 
The apparent heat capacity is calculated as $C_{a p p}=\rho_{\text {soil }} C_{\text {soil }}-\rho_{i c e} L_{f} \frac{\partial \theta_{i c e}}{\partial T}$ to express both the heat conduction and latent heat (phase change) terms. On the basis of the assumption of zero ice gauge pressure and osmotic pressure, Clausius-Clapeyron equation is utilized to convert $-\rho_{i c e} L_{f} \frac{\partial \theta_{i c e}}{\partial T}$ into $\rho_{i c e} \frac{\left(L_{f}^{2}\right)}{g T} \frac{d \theta}{d \varphi}$. This is a general procedure to derive the apparent heat capacity and it is independent of which form of the soil-freezing characteristic function is used. The assumption and the Clausius-Clapeyron equation used in Dall'Amico (2011) is the same as Hansson et al. (2004) (i.e., $\frac{d P}{d T}=\frac{L_{f}}{V_{w} T}$ ), there is no difference between these two papers in calculating the apparent heat capacity. The beauty of the equation (19) - (21) in Dall'Amico et al., (2011) - the equations used to estimate the additional freezing pressure due to the unfrozen water pressure - is that they are fully energy conservative (contrary to other formulations) and are also independent of the specific hydraulic parameterization of the soil (even though later on van Genuchten method is used in Dall'Amico et al. 2011). As a matter of fact, in T\&CFT, three different soil hydraulic parameterizations can be used: (i) van Genuchten, (ii) Saxton-Rawls and (iii) Clapp and Hornberger. For all of these the liquid water pressure is derived with equation (21), but then the actual liquid and frozen water content depend on the specific soil hydraulic parameterization chosen. Before implementing the Dall'Amico et al., (2011) formulation, we had a lot of problems related to energy conservation in the freezing/melting phases, problems that were not apparent for a week-ten days of simulations but developed over time, so we checked carefully that energy is indeed properly conserved in the long-term in T\&C-FT.

Changes in the manuscript: "The soil freezing characteristic curve providing the liquid water potential in a frozen soil is computed following the energy conservative solution proposed by Dall'Amico et al. (2011) and it can be combined with various soil hydraulic parameterizations including van Genuchten and Saxton and Rawls to compute the maximum liquid water content at a given temperature and consequently ice and liquid content profiles at any time step (Fuchs et al., 1978;Yu et al., 2018)."

14. Section 2.6: I think this section lacks some important clarifications. First, I think the 
differences between the models are more complicated than coupled/uncoupled. The vadose zone equations of T\&C model are not coupled, because the water and heat equations are independent from each other. On the other end, the heat equation of STEMMUS needs to be coupled to the mass transfer equation because of the consideration of different processes or constituents such as heat advection. However, when adding the freezing/thawing processes, the heat and water equations of T\&C becomes somehow coupled (at least one-way) due to the temperature dependency of the hydraulic conductivity. It is unclear if the authors used the names unCPLD, unCPLD-FT and CPLD for their models to characterize the way the heat and water equations are solved or to characterize how the two components (T\&C and STEMMUS) are used. If it is the former, I suggest that they authors rename their simulations as T\&C, T\&C-FT and T\&C-STEMMUS. In any case, the coupling characteristics of the different simulations should be further explained. Secondly, it is unclear which processes/parameters are considered in each simulation. For example, the authors state in the discussion that "unCPLD-FT simulation accounting for soil-freezing in a simplified way in comparison to STEMMUS (e.g., the CPLD simulation)" (line 418-419). However, to my knowledge, the difference in the way STEMMUS accounts for soil-freezing processes is never explained. A paragraph describing the different processes that each simulation is accounting for is necessary in this section. This can also take the form of a table.

Response: Thanks a lot for the comment. Yes, the "coupling" in the label was referring to the coupling among models rather than in the processes. In order to present the difference among the model versions and clarify the words 'unCPLD, CPLD', we added Table 1 and relevant descriptions in the Sect. 3.5 to explain the difference and the processes considered in each simulation case.

15. Figure 3: The grey line is hard to distinguish. I suggest making it slightly darker or thicker.

Response: We made the grey line darker and thicker in Figure 4. 
16. Figures 5 and 6: It looks like deeper soil moisture and temperature is not well reproduced by any of the models, even though it is not discussed in the text. This has the consequence of poorly representing ice content (Figure 7), mostly in 2017-2018. It is understandable considering the model has not been calibrated and the goal of this manuscript, which focuses more on the growing season, is not necessarily to validate the models. However, I think this poor fit with field measurement should be discussed in the text. There are many reasons that could explain this, such as the presence of heterogeneity in the soil or of freezing-point depression due to increased salinity.

Response: In Sect. 4.2, We now discussed the reasons for the discrepancies between the model simulated and observed soil moisture and temperature at deeper soil layers.

Changes in the manuscript: "It should be noted that for the deeper soil layers (e.g., $60 \mathrm{~cm}$ in Figure 7), all models tended to simulate the early start of freezing soil temperatures and considerably underestimated the soil temperature during the frozen period. This can be due to the uncertainties in soil organic layer parameters, the not fully captured snow cover effect (Gouttevin et al., 2012), a potentially pronounced heterogeneity in soil hydrothermal properties, or the potential role of solutes on the freezing-point depression (the presence of solute lowers the freezing soil temperature) (Painter and Karra, 2014). These mismatches in deep soil temperature degraded the model performance in simulating the dynamics of liquid water (Figure 6) and ice content (Figure 8) during the frozen period."

17. L 303: I think they authors meant "unCPLD-FT" instead of "unCPLD".

Response: We initially used "unCPLD" model simulations to refer to both the "unCPLD" and "unCPLD-FT" model simulations, as Fig. 8 also presents the water flow simulations of unCPLD model. To avoid confusion, we rephrased this sentence as"The time-series of soil ice content and water flux from unCPLD, unCPLD-FT and CPLD model simulations for soil layers below $2 \mathrm{~cm}$ are presented in Figure 8."

Printer-friendly version

18. L 307-309: I do not agree that the CPLD model shows a good match with field 
measurements of ice content, at least not as currently showed in winter 2017-2018.

Response: Yes. Both the unCPLD and CPLD model cannot well reproduce the soil ice content based on current simulations. Here, we would like to stress the difference between unCPLD-FT and CPLD model. CPLD model presents a shallower freezing ice depth compared to unCPLD-FT model. We attributed this to the physical difference between unCPLD-FT and CPLD model, i.e., the constraints by the interdependence of liquid, ice, vapor, air components in the soil pores are considered by CPLD model.

Changes in the manuscript: "It is to note that compared to unCPLD-FT model, CPLD model presented a relatively lower presence of soil ice content, while its temporal dynamics was closer to the observed freezing/thawing front propagation. The difference between the two simulations can be attributed to the constraints imposed by the interdependence of liquid, ice and vapor in the soil pores that is considered only in CPLD model."

19. Figure 8: Please define acronyms in the caption (e.g., "(a) Gross Primary Production" instead of "(a) GPP”)-friendly version

Response: We describe acronyms in the caption of Figure 9.

Changes in the manuscript: "Figure 9. Comparison of observations from Eddy Covariance (OBS) or MODIS remote sensing and simulated (a) Gross Primary Production (GPP), (b) Leaf Area Index (LAI), (c) Net Ecosystem Exchange (NEE), and (d) Ecosystem respiration (Reco) using unCPLD, unCPLD-FT, and CPLD model. MODIS refers to the data from MODIS-GPP and MODIS-LAI products."

20. Figure 9: The authors use $R 2$ here and $R$ in Figure 8 . I suggest them to choose one and be consistent.

Printer-friendly version

Response: Yes, sorry for the confusion. We use the determination coefficient R2 and keep it consistent in Figure 5 and Figure 10.

Discussion paper

21. Figure 10: Please define acronyms in the figure caption. 
Response: We added the descriptions of the acronyms as " $T_{v}$, transpiration; $E_{s}$, surface evaporation; $E_{I N}$ and $E_{S N}$, evaporation from intercepted canopy water and snow cover; $\Delta V s$, changes in soil water storage; $L_{K}$, deep leakage water.".

\section{L 381-382: Confusing sentence. Should we compare to unCPLD or unCPLD-FT?}

Response: Here we compare unCPLD model simulations with unCPLD-FT model simulation to highlight the role of ice content and latent heat associated with phase change only. This has been clarified as"Less amount of water was consumed by ET from unCPLD-FT simulations than that from unCPLD."

23. L 383-384: What could explain cooler late winter temperatures in unCPLD-FT? Latent heat slowing down the thawing? Lower bulk heat capacity of frozen soil? Please provide hypotheses.

Response: As for unCPLD-FT model, the effect of ice content and latent heat due to phase change were taken into account, while these two effects were absent by unCPLD model. During the late winter (mainly thawing periods), the thawing process was retarded by the heat absorption due to phase change. Secondly, the ice induced soil heat capacity also damped the magnitude of temperature variations, make the thawing process of soil temperature more difficult and slower. Generally, as discussed earlier there is a quite evident cryoscution in the unCPLD-FT simulation that generates a considerable amount of ice content in the soil that takes time to be melt and reduce the average temperature. Thus there are cooler late winter temperatures in the unCPLDFT model than in the unCPLD simulations.

Changes in the manuscript: "The cooler late winter temperatures from unCPLD-FT simulations can be attributed to the retardation of the thawing process due to the phase change-induced heat absorption and the soil ice-induced modification of bulk heat capacity during the freezing-thawing transition period, which damped the magnitude of temperature variations and delayed the thawing process." 
24. L 396-400: This analysis could be improved. The coupling is not the only difference between CPLD and unCPLD-FT. STEMMUS is simulating some subsurface processes that T\&C does not (e.g., heat advection, air flow, vapor flow). I recommend providing a more detailed analysis then simply justifying the differences by the coupling. Also, how is ice content and hydraulic conductivity being simulated differently in CPLD than in unCPLD-FT?

Interactive

Response: We agree with your points that the coupling is not the only difference between CPLD and unCPLD-FT model. These contexts (L396-400) were used to explain why the water storage amount in the vadose zone is increased while the bottom leakage decreased for CPLD model (Figure 11). It lies in the difference of the considered subsurface processes between CPLD and unCPLD models (Sect. 3.5, Table 1). We further described the hydraulic conductivity (and its temperature dependency), vapor density in supplement S1.

Hydraulic conductivity in CPLD (T\&C-STEMMUS) model is dependent on temperature in two ways. 1. The impedance effect of soil ice content on the hydraulic conductivity , which is dependent on soil temperature, (i.e., reducing the saturated hydraulic conductivity via an empirical impedance factor). 2 . The water viscosity effect on hydraulic conductivity. As the temperature decreases, water movement slows down.

Changes in the manuscript: "We attribute this to the way ice content is simulated in the CPLD simulation, and also to the temperature dependence of soil hydraulic conductivity (see Table 1 and Supplement S1). Specifically, the high accumulation of ice content in the unCPLD-FT simulations indicates a relatively stronger cryosuction effect than in CPLD simulations. This cryosuction effect is mitigated in the fully coupled model because of water vapor transfer and thermal gradients, even though different solutions in the parameterization of bulk soil thermal conductivity and volumetric soil heat capacity could also be responsible for the difference. Overall, taking into account the fully coupled water and heat physics modify the temporal dynamics of ice formation and thawing in the soil and activates temperature effects on water flow (i.e., low soil

Printer-friendly version

Discussion paper

\section{C15}


25. L 407-410: There are two requirements to experience heat advection: water flow and difference in temperature. While the former is shown in Figure 7, there is no evidence shown for the latter. It would be interesting if the authors could provide some evidence (can be with numbers or words) that heat advection (or convective heat) is mostly relevant during the frozen period.

Response: Here, we highlight the difference between unCPLD and CPLD models on heat advection effects. As shown in Figure $8 \mathrm{c}$ and d/e, the difference in water flow can be several orders of magnitude. We added some references here to corroborate this point.

Changes in the manuscript: "The liquid water flux-induced convective heat flux is mostly relevant during the frozen period (Boike et al., 2008;Kane et al., 2001;Yu et al., 2020). As it has been observed, a certain amount of liquid water/vapor flux moving toward the freezing front and this effect is different between unCPLD-FT and CPLD while absent in unCPLD (Figure 8). For the unfrozen period, instead, the total mass fluxes were comparable between the two unCPLD and CPLD simulations. For the temperature gradient, there is not much difference between unCPLD and CPLD simulations during both the growing season and frozen period."

26. L 413: I think Figure 8 should be referred here instead of Figure 9.

Response: Yes sorry. We corrected it in the manuscript (Line 473). Thanks a lot. 
Boike, J., Hagedorn, B., and Roth, K.: Heat and Water Transfer Processes in Permafrost Affected Soils: A Review of Field and Modeling Based Studies for the Arctic and Antarctic, Plenary Paper, Proceedings of the 9th International Conference on Permafrost, University of Alaska, Fairbanks, USA, 2008.

Boone, A., Masson, V., Meyers, T., and Noilhan, J.: The Influence of the Inclusion of Soil Freezing on Simulations by a Soil-Vegetation-Atmosphere Transfer Scheme, J Appl Meteorol, 39, 1544-1569, 10.1175/1520-0450(2000)039<1544: tiotio > 2.0.co; , 2000.

Cheng, G., and Wu, T.: Responses of permafrost to climate change and their environmental significance, Qinghai-Tibet Plateau, Journal of Geophysical Research: Earth Surface, 112, 10.1029/2006JF000631, 2007.

Dall'Amico, M., Endrizzi, S., Gruber, S., and Rigon, R.: A robust and energy-conserving model of freezing variably-saturated soil, Cryosphere, 5, 469-484, 10.5194/tc-5-4692011, 2011.

Flerchinger, G. N., and Saxton, K. E.: Simultaneous heat and water model of a freezing snow-residue-soil system. I. Theory and development, Transactions of the American Society of Agricultural Engineers, 32, 565-571, 1989.

Hansson, K., Šimůnek, J., Mizoguchi, M., Lundin, L. C., and van Genuchten, M. T.: Water flow and heat transport in frozen soil: Numerical solution and freeze-thaw applications, Vadose Zone J, 3, 693-704, 2004.

Kane, D. L., Hinkel, K. M., Goering, D. J., Hinzman, L. D., and Outcalt, S. I.: Nonconductive heat transfer associated with frozen soils, Global Planet Change, 29, 275292, 10.1016/S0921-8181(01)00095-9, 2001.

Painter, S. L.: Three-phase numerical model of water migration in partially frozen ge- 
ological media: Model formulation, validation, and applications, Comput Geosci, 15, 69-85, 10.1007/s10596-010-9197-z, 2011.

Qin, Y., Lei, H., Yang, D., Gao, B., Wang, Y., Cong, Z., and Fan, W.: Longterm change in the depth of seasonally frozen ground and its ecohydrological impacts in the Qilian Mountains, northeastern Tibetan Plateau, J Hydrol, 542, 204-221, https://doi.org/10.1016/j.jhydrol.2016.09.008, 2016.

Wang, L., Zhou, J., Qi, J., Sun, L., Yang, K., Tian, L., Lin, Y., Liu, W., Shrestha, M., Xue, Y., Koike, T., Ma, Y., Li, X., Chen, Y., Chen, D., Piao, S., and Lu, H.: Development of a land surface model with coupled snow and frozen soil physics, Water Resour Res, 53, 5085-5103, 10.1002/2017WR020451, 2017b.

Wang, L., Liu, H., Shao, Y., Liu, Y., and Sun, J.: Water and CO2 fluxes over semiarid alpine steppe and humid alpine meadow ecosystems on the Tibetan Plateau, Theor Appl Climatol, 131, 547-556, 10.1007/s00704-016-1997-1, 2018.

Wang, X., Yi, S., Wu, Q., Yang, K., and Ding, Y.: The role of permafrost and soil water in distribution of alpine grassland and its NDVI dynamics on the Qinghai-Tibetan Plateau, Global Planet Change, 147, 40-53, 10.1016/j.gloplacha.2016.10.014, 2016.

Yu, L., Zeng, Y., Wen, J., and Su, Z.: Liquid-Vapor-Air Flow in the Frozen Soil, Journal of Geophysical Research: Atmospheres, 123, 7393-7415, 10.1029/2018jd028502, 2018.

Yu, L., Zeng, Y., and Su, Z.: Understanding the Mass, Momentum and Energy Transfer in the Frozen Soil with Three Levels of Model Complexities, Hydrol Earth Syst Sci Discuss, 2020, 1-24, 10.5194/hess-2020-253, 2020.

Zhao, L., Li, J., Xu, S., Zhou, H., Li, Y., Gu, S., and Zhao, X.: Seasonal variations in carbon dioxide exchange in an alpine wetland meadow on the Qinghai-Tibetan Plateau, Biogeosciences, 7, 1207-1221, 10.5194/bg-7-1207-2010, 2010.

Zheng, D., Velde, R. v. d., Su, Z., Wen, J., Wang, X., and Yang, K.: Evaluation of 
Noah Frozen Soil Parameterization for Application to a Tibetan Meadow Ecosystem, J Hydrometeorol, 18, 1749-1763, 10.1175/jhm-d-16-0199.1, 2017.

Interactive

comment 


\section{Tables and Figures}

Table 1. Numerical experiments with various mass and energy transfer processes

\begin{tabular}{|c|c|c|c|}
\hline \multirow[b]{2}{*}{ Experiment } & \multicolumn{2}{|c|}{ Soil Physical Processes } & \multirow[b]{2}{*}{ Model Components } \\
\hline & Unfrozen period & Unfrozen period & \\
\hline unCPLD & independent WHT & $\begin{array}{l}\text { independent WHT; } \\
\text { no ice effect; } \\
\text { no LH due to phase change }\end{array}$ & $\begin{array}{l}\text { T\&C } \\
\text { (Eqs. } 1 \text { \& 3) }\end{array}$ \\
\hline unCPLD-FT & independent WHT & $\begin{array}{l}\text { FT induced WHT coupling; } \\
\text { ice effect; } \\
\text { LH due to phase change }\end{array}$ & $\begin{array}{l}\text { T\&C-FT } \\
\text { (Eqs. } 1 \& \text { 4) }\end{array}$ \\
\hline CPLD & tightly coupled WHT & $\begin{array}{l}\text { tightly coupled WHT; } \\
\text { ice effect; } \\
\text { LH due to phase change; } \\
\text { CH due to liquid/vapor flow }\end{array}$ & $\begin{array}{l}\text { T\&C-STEMMUS } \\
\text { (Eqs. } 7 \& \text { \&) }\end{array}$ \\
\hline
\end{tabular}

Note: Independent WHT, Soil Water and Heat Transfer process is independent.

FT induced WHT coupling, Soil Water and Heat Transfer process is coupled only during the freezing/thawing (FT) period. Soil water flow is affected by temperature due to the temperature dependency of hydraulic conductivity (the impedance effect as the presence of soil ice content). Tightly coupled WHT, Soil Water and Heat Transfer process is tightly coupled; vapor flow, which links the soil water and heat flow, is taken into account; thermal effect on water flow is considered (the hydraulic conductivity and matric potential is dependent on soil temperature; when soil freezes, the hydraulic conductivity is reduced by the presence of soil ice, which is temperature dependent); the convective/advective heat due to liquid/vapor flow can be calculated.

Ice effect, the explicit simulation of ice content and its effect on the hydraulic/thermal

Interactive

comment 


\section{properties.}

LH due to phase change, latent heat due to the phase change. $\mathrm{CH}$ due to liquid/vapor flow, convective heat due to liquid/vapor flow.

Figure 1. Geographical location of Maqu soil moisture/temperature (SMST) monitoring network and the Centre station.

Figure 2. Observed cumulative precipitation (P) and evapotranspiration (ET) (a) and observed propagation of freezing/thawing front (FTFP), with the blue and red color for the propagation of freezing front and thawing front (FFP \& TFP), respectively (b) for the period 25 Mar. 2016- 12 Aug. 2018 at Maqu site.

Figure 4. Comparison of observed and simulated 5-day moving average dynamics of net radiation $(\mathrm{Rn})$, latent heat flux (LE), and sensible heat flux $(\mathrm{H})$ using the original (uncoupled) T\&C (unCPLD), T\&C with consideration of FT process (unCPLD-FT) and coupled T\&C and STEMMUS (CPLD) model.

Figure 5. Scatter plots of observed and model simulated daily average surface fluxes (net radiation: $\mathrm{Rn}$, latent heat: $\mathrm{LE}$ and sensible heat flux: $\mathrm{H}$ ) using the original (uncoupled) T\&C (unCPLD), T\&C with consideration of FT process (unCPLD-FT) and coupled T\&C and STEMMUS (CPLD) model, with the color indicating the frequency of surface flux values.

Please also note the supplement to this comment: https://tc.copernicus.org/preprints/tc-2020-88/tc-2020-88-AC2-supplement.pdf 
Interactive comment on The Cryosphere Discuss., https://doi.org/10.5194/tc-2020-88, 2020.

Interactive

comment 

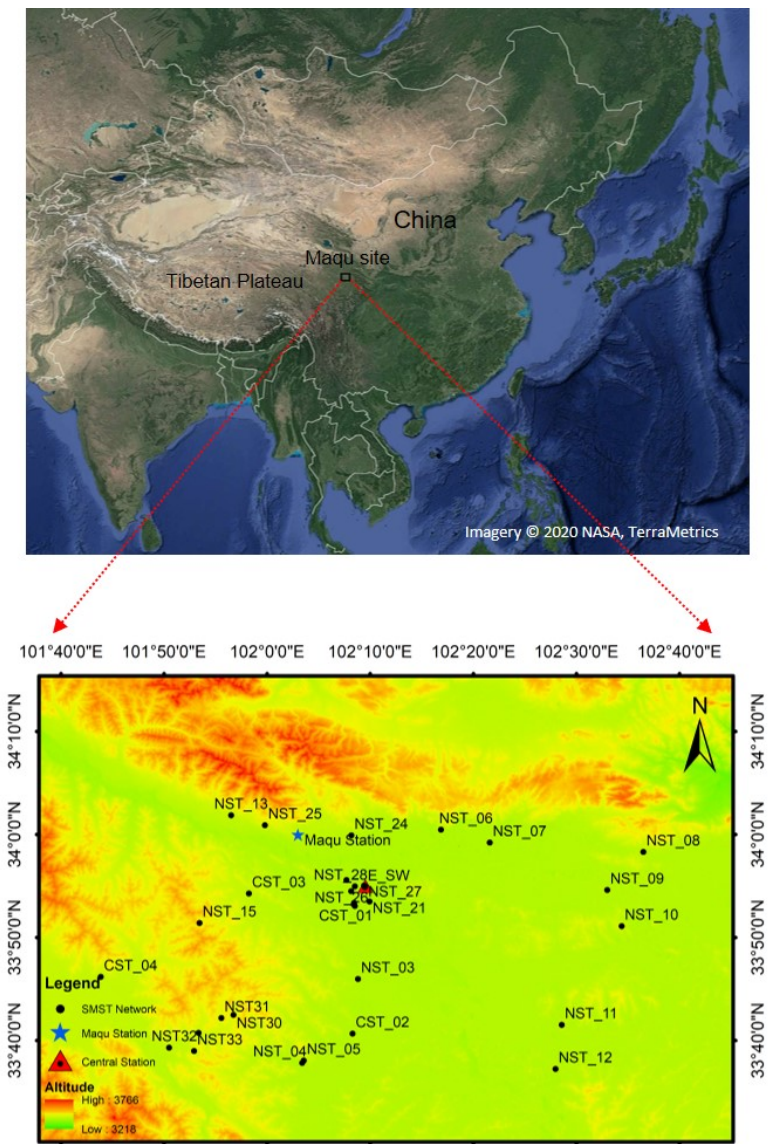

Interactive

comment

Fig. 1. Figure 1. Geographical location of Maqu soil moisture/temperature (SMST) monitoring network and the Centre station. 
(a)

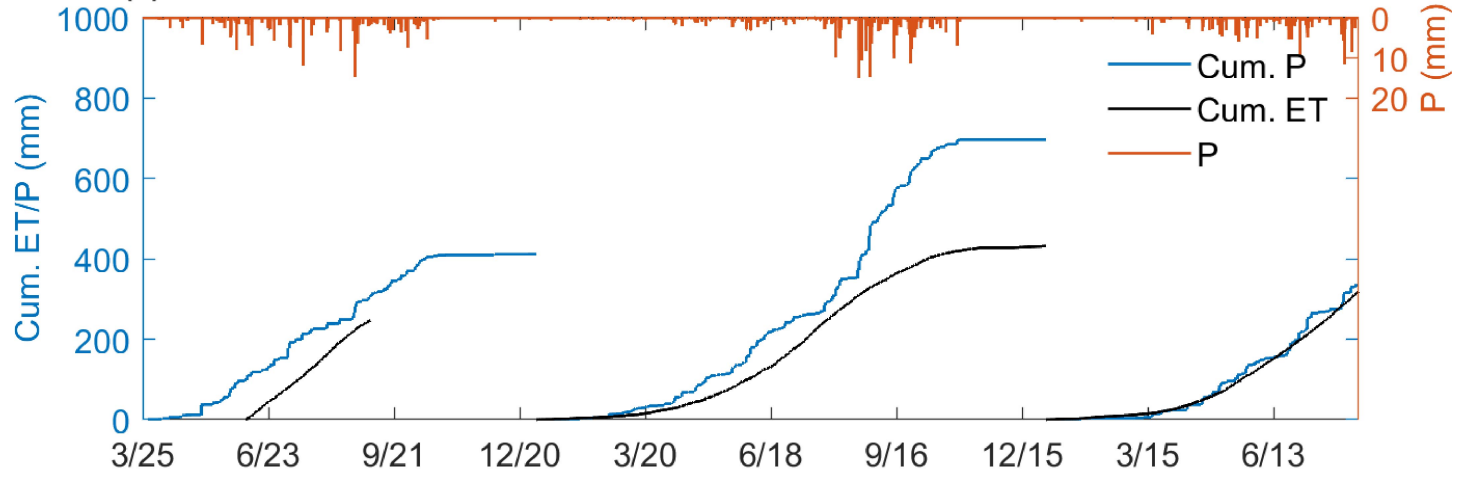

(b)

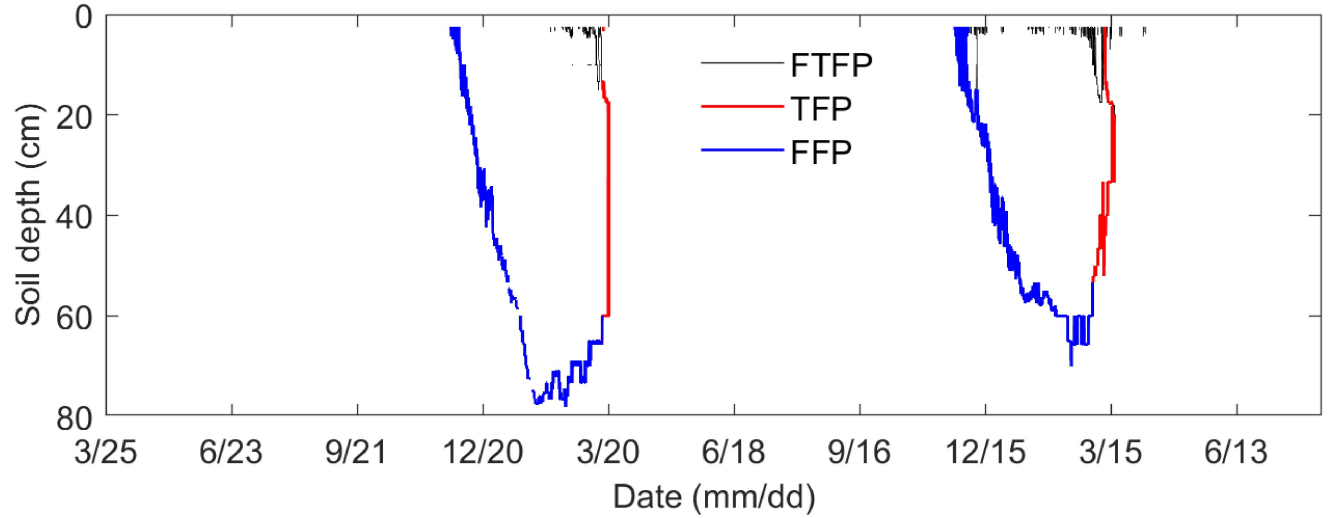

Fig. 2. Figure 2. Observed cumulative precipitation $(P)$ and evapotranspiration (ET) (a) and observed propagation of freezing/thawing front (FTFP)(b) for the period 25 Mar. 2016- 12 Aug. 2018 at Maqu site.
TCD

Interactive

comment
Printer-friendly version

Discussion paper 

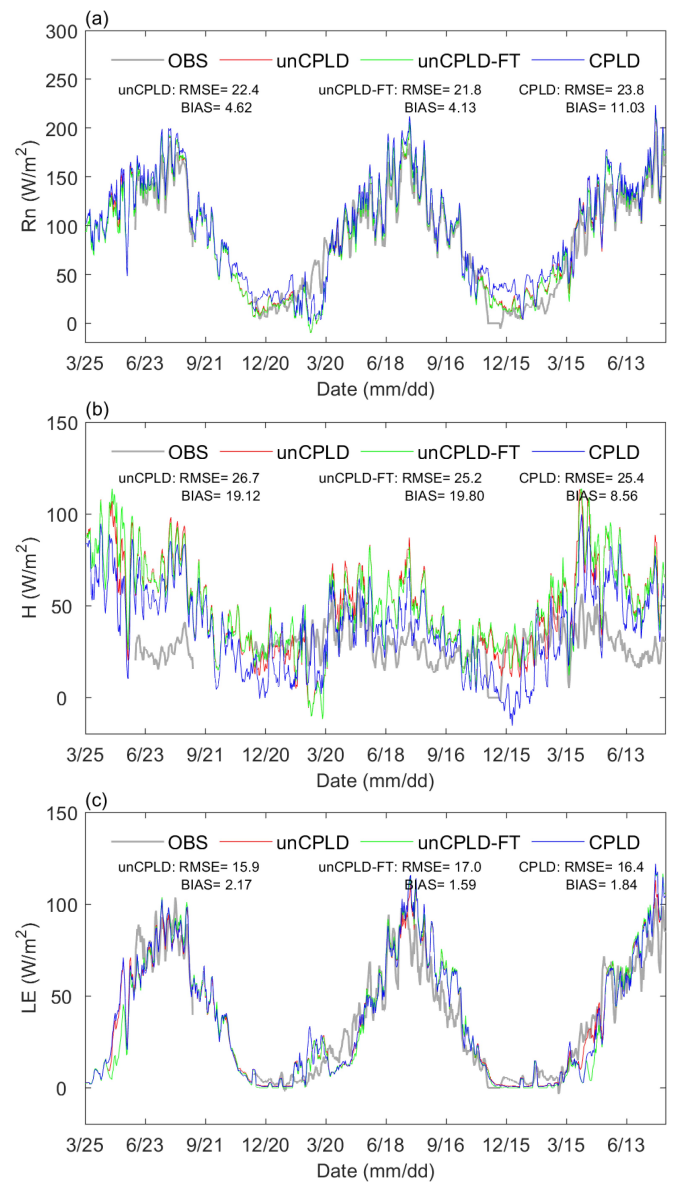

Interactive

comment

Fig. 3. Figure 4. Comparison of observed and simulated 5-day moving average dynamics of net radiation $(\mathrm{Rn})$, latent heat flux (LE), and sensible heat flux $(\mathrm{H})$ using unCPLD, unCPLD-FT and CPLD model. 
unCPLD
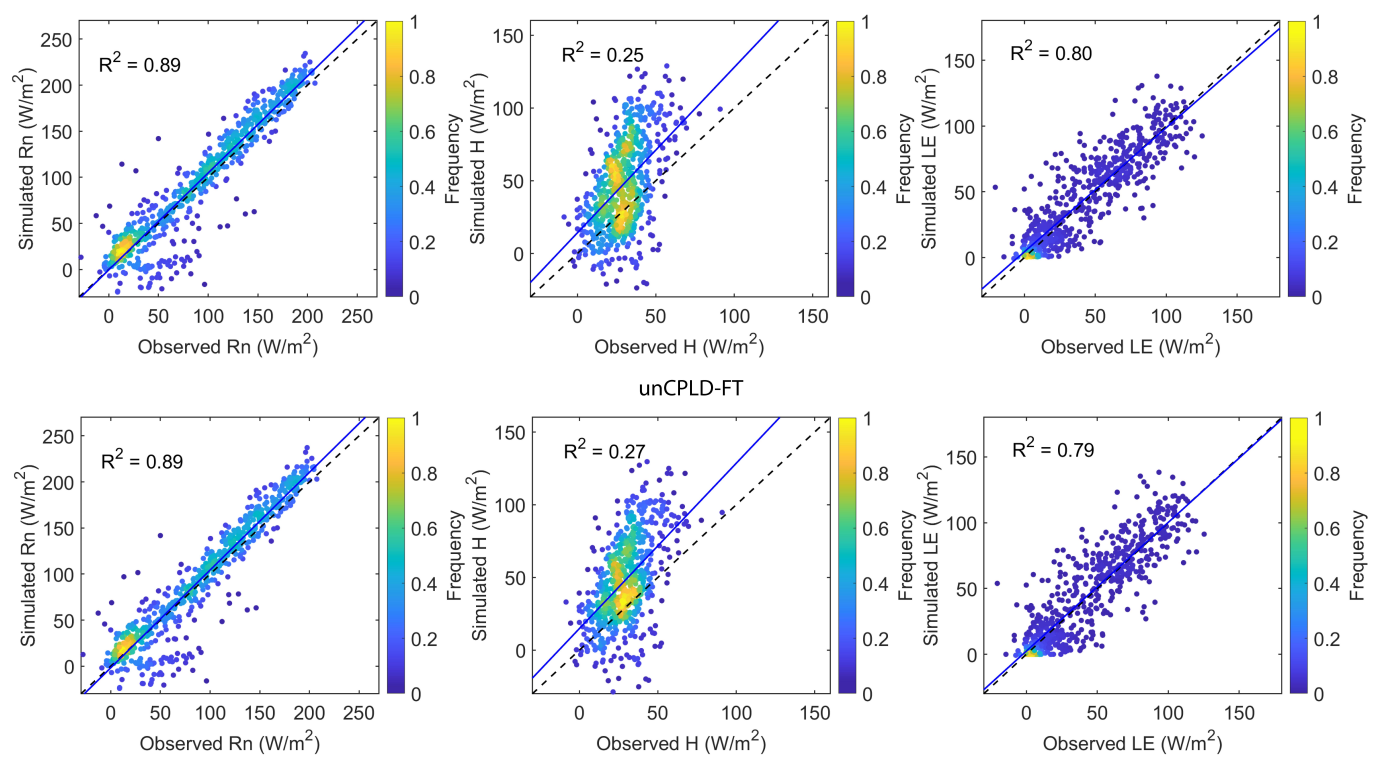

CPLD
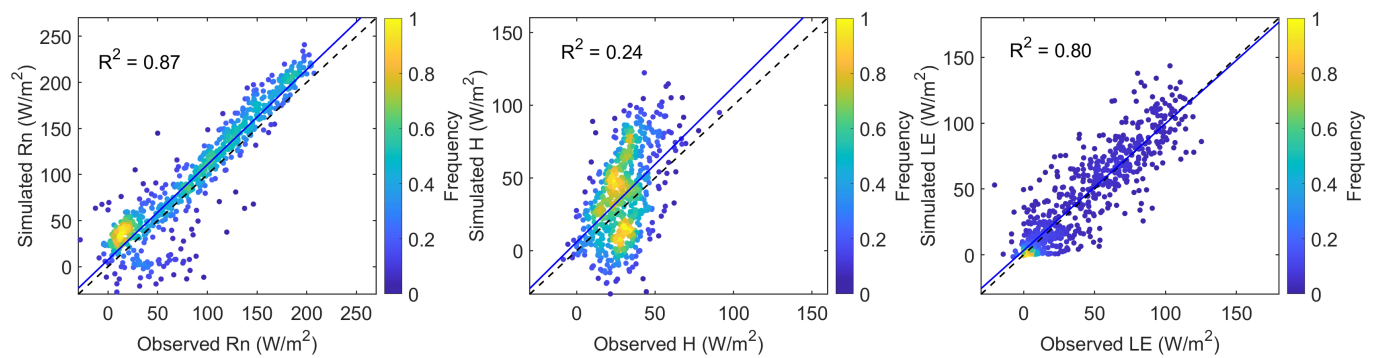

Interactive

comment

Fig. 4. Figure 5. Scatter plots of observed and model simulated daily average surface fluxes (net radiation: $\mathrm{Rn}$, latent heat: LE and sensible heat flux: H) using unCPLD, unCPLD-FT and CPLD model. 